\title{
SUR LA TAXONOMIE \\ DE QUELQUES ESPECES D'ECDYONURUS DU SUD-OUEST DE LA FRANCE \\ [Ephemeroptera]
}

\author{
par A. Thomas.
}

L'identification des espèces du genre Ecdyonurus ne peut être effectuée avec certitude qu'en Grande-Bretagne. Grâce aux travaux de Kimmins [1942, 1954] et de Macan [1949], les trois espèces E. venosus (Fabricius, 1775), E. dispar (Curtis, 1834) et $E$. torrentis Kımmins, 1942 sont maintenant bien connues.

La situation est beaucoup moins claire en Europe continentale, en partie en raison d'un nombre d'espèces plus élevé. Aussi était-il souhaitable de comparer mon propre matériel, récolté dans le SudOuest de la France, à des exemplaires de Grande-Bretagne. Grâce à un envoi du $D^{r}$ T. T. MAcaN, il a été possible d'effectuer cette comparaison pour les trois stades larvaire, subimaginal et imaginal.

Je prie le $D^{r}$ Macan d'accepter mes plus vifs remerciements, ainsi que Mile Kelner-Pillault (Muséum National d'Histoire Naturelle de Paris), R. P. Delattre (Colegio del Salvador, Saragosse), MM. Español (Museo de Zoologia, Barcelone), Kimmins (British Museum, Londres), Nicolau-GuilIAUMet (Laboratoire Arago, Banyuls), Régnier (Muséum d'Histoire Naturelle, Genève), Thibault (Station d'Hydrobiologie Continentale, Biarritz) qui m'ont permis d'examiner des exemplaires de leur collection personnelle ou de celle de leur établissement. L'aide et les conseils de M. C. BerthéLEMY m’ont été précieux.

L'examen de mon matériel, qui comprend plus d'un millier d'imagos et de subimagos des deux sexes, souvent accompagnés de leur exuvie larvaire, montra qu'il existait au moins 7 espèces d'Ecdyonurus dans le Sud-Ouest de la France. Deux d'entre elles sont représentées par un nombre insuffisant d'individus et ne seront pas envisagées dans cette note. Les 5 autres peuvent être réparties en trois ensembles en fonction de la forme du pénis : $E$. dispar et $E$. pazsiczkyi possèdent des lobes latéraux courts, $\boldsymbol{E}$. venosus et $\boldsymbol{E}$. forcipula des lobes plus allongés, $\boldsymbol{E}$. angelieri n. sp., enfin, des sclérites internes de forme caractéristique.

Avant d'aborder l'étude des critères de différenciation de ces espèces à l'état imaginal et à l'état larvaire seront exposées les 
raisons de l'emploi du nom «Ecdyonurus pazsiczkyi » pour désigner la «forme claire d'E. fluminum» décrite par Eaton [1887].

\section{1. - LE PROBLEME E. FLUMINUM [PICTET, 1844]}

Ecdyonurus dispar fut décrit par Curtis en 1834. Le nom tomba dans l'oubli jusqu'en 1942, où Krmmins sépara définitivement cette espèce d'E. venosus et d'une nouvelle espèce britannique, $E$. torrentis. E. dispar, cité des Pyrénées par Kimmıss [1942], figure dans les travaux de Grand [1953, 1960], Bogoescu [1958], Landa [1959] et Fontaine [1964]. L'espèce figurée par Grand est en fait un Ecdyonurus du groupe helveticus, sans doute E. zelleri [EATon, 1885 ${ }^{1}$. En 1953, Grand précisa d'ailleurs que les exemplaires étudiés étaient dépourvus de pattes antérieures.

La situation est plus confuse pour $E$. fluminum. L'espèce fut décrite par Picter en 1844, d'après des exemplaires capturés près du Rhône en amont et en aval du lac Léman. Dès 1871, il ne subsistait plus de la série type que des subimagos [EATON, 1871 ]. EAton [1883-1889] redécrivit $E$. fluminum, en y ajoutant une «variété claire» qui ne correspond pas à la description originale de Pictex. Le matériel d'Eaton fut revu par Kimmins [1942] qui a bien voulu m'en communiquer quelques exemplaires. La « variété sombre» et la «variété claire» d'EAToN appartiennent à deux espèces bien distinctes par les genitalia comme par la coloration. Toutes deux diffèrent également d'E. dispar.

Les exemplaires de la «variété sombre» de la collection MAC. Lachlan du British Museum, déterminés par Eaton, proviennent du lac Léman à Genthod et non de la localité typique. Leurs genitalia ne diffèrent pas de façon significative de ceux d'E. forcipula figurés par Kmmins [1942]. Kımmins (ibid.) avait proposé à titre provisoire un critère de différenciation basé sur la coloration des tergites abdominaux. L'examen d'un nombre important d'E. forcipula a montré que les exemplaires de Genthod déterminés par EATon, ainsi qu'un exemplaire de même origine conservé au Muséum de Genève, rentraient dans le cadre des variations d'E. forcipula. Aucun auteur autre qu'Eaton ou Kimmins ne semble avoir adopté l'interprétation précédente, selon laquelle $E$. fluminum serait une forme très proche d'E. forcipula, ou même, comme je le pense, une forme qui rentre dans les limites de variation de cette espèce.

La «variété claire» d'EATon, par contre, fut désignée sous le nom «E. fluminum»par Grandi [1953, 1960] et Fontaine [1964]. 
J'ai retrouvé cette espèce dans une des localités citées par EAToN, la Garonne près de Toulouse.

Le nom «E. fluminum $»$ fut encore attribué à une troisième espèce. D'après Kımmins [1942], Ulmer détermina comme « $E$. fluminum » des $E$. dispar britanniques. Cette détermination d'ULMER est en accord avec sa description [1929], avec les figures de genitalia du même auteur ainsi que de Schoznemund [1930] et de Bogoescu et Tabacaru [1962].

Trois espèces au moins ont donc été désignées par le nom d'E. fluminum : $E$. forcipula par EATon et Kimmins, $E$. dispar par Ulmer, Schoenemund et Bogoescu et Tabacaru, et la «variété claire»d'Eaton par Eaton lui-même, Grandi et Fontaine.

Le regretté H. Gisin, du Muséum d'Histoire Naturelle de Genève, a bien voulu m'indiquer que la série type de Prctet n'existait plus. M. RÉGNiER, préparateur au même établissement, a récolté à mon intention des Ecdyonurus dans la localité typique de Picter, le Rhône en aval de Genève. Les individus, obtenus par élevage, sont conformes à la description et aux planches en couleurs de PIctet. Ce sont des Ecdyonurus dispar [Cuntis, 1834]. Il ne peut être exclu qu'E. forcipula, capturé près du lac Léman, vive aussi dans le Rhône, ou qu'il y ait vécu du temps de Picter, ni même que cet auteur ait confondu les deux espèces. Mais la description originale et les figures de Picter, en l'absence de type, peuvent seules servir de référence. Cette description et ces figures correspondent mieux à $E$. dispar qu'à $E$. forcipula. En outre, la synonymie $E$. fluminum $=E$. forcipula introduirait plus de confusion encore dans la nomenclature.

Aussi les synonymies suivantes sont-elles proposées :

Ecdyonurus fluminum PICTET, 1844], Ulmer [1929], SchoeneMUND [1930] fig. $25=$ E. dispar [CuRtis, 1834],

Ecdyonurus fluminum EATON [1883] pro parte (variété sombre), Kimmins [1942], nec Pictet [1844] = E. forcipula [Pictet, 1844].

Reste la question de savoir comment doit être désignée la « variété claire»d'EAToN, qui ne correspond nullement à la description et aux figures de Picter.

ILlIEs [1967] a proposé la synonymie E. pazsinczkyi (=E. pazsi$c z k y i$, lapsus calami) $=E$. fluminum. D’après la description de Pongracz [1913], E. pazsiczki ne diffère de la «variété claire» que par des dét-ils mineurs : les «gonopodes noirs» sont gris plus ou moins foncé sur les exemplaires de la Garonne et les taches latérales obliques noires "en forme de baguette » semblent également un peu plus larges sur le dessin de Pongracz. La synonymic proposée par ILliEs a donc été adoptée ici, sous réserve d'une confirmation ou d'une infirmation après examen des types de Pongracz ou d'exemplaires provenant d'une des localités typiques. 


\section{2. - ECDYONURUS PAZSICZKYI PONGRACZ, 1913 ET E. DISPAR [CURTIS, 1834]}

\section{1. - Matériel examiné :}

- E. pazsiczkyi : outre quatre exemplaires d'Eaton, j'ai pu étudier la collection Despax, conservée au Muséum de Paris et ma propre collection, en tout une vingtaine d'individus provenant tous de la Garonne soit à Toulouse (A. E. Eaton leg., R. Despax leg.) soit à $5 \mathrm{~km}$ en amont, à Portet-sur-Garonne (4-VI1932 : R. Despax leg., 26-IV-1966 : A. T. leg.) soit à $30 \mathrm{~km}$ en aval, à Verdunsur-Garonne (17-VII-1967 : A. T. leg.). De nombreuses nymphes ont de plus été capturées.

- E. dispar : plus de cent imagos $\delta$, dont un tiers accompagné des exuvies larvaires, provenant des départements suivants :

Tarn-et-Garonne : la Garonne à Verdun-sur-Garonne (20-VII-1967 : A. T. leg.). Ariège : le Volp (15-25-VII-1963 et 9-14-VIII-1963 : C. BERTHÉL.eMY leg.; 5-VI à 28-VIII-1967 : A. 'T. leg.); l'A rize à $300 \mathrm{~m}$ (14-21-X-1963, 21-VII-1965 : C. BERThélemy leg.); le Lez à $430 \mathrm{~m}$ (7-10-VII-1963 : C. Berthélemy leg.).

Tarn : la Vère à Mauriac (26-V-1961: H. Décamps leg.).

J'ai également pu examiner 6 individus $\sigma$ provenant du Rhône à ChancyLalaire (Suisse, 14-17-VII-1967 : J.-C. Rḱgnıs leg.) et deux imagos o britanniques du lac Windermere (Westmorland, 2-VII-1947) et de la rivière Eden (Cumberland, 19-VIII-1947) ('T. T. MaCAN leg.).

\section{2. - Comparaison des deux espèces.}

\section{Adultes.}

Coloration. - La coloration semble assez constante chez E. pazsiczkyi, mais est très variable chez $E$. dispar.

Tête : chez E. pazsiczkyi, la tête est jaune légèrement rosâtre; elle est plus foncée chez $E$. dispar. L'anneau de pigment brun violet qui entoure la base de chaque ocelle apparaît plus nettement chez E. pazsiczkyi. Chez les deux espèces, les yeux sont d'un gris tirant sur le violet mais nettement plus violacés chez $\boldsymbol{E}$. pazsiczkyi.

Thorax :

- chez E. pazsiczkyi, le thorax est dorsalement brun très clair, un peu plus clair latéralement, et à peine plus foncé ventralement. Une tache allongée, brun-violacée, très foncée, s'étend de l'extrémité de la nervure costale jusqu'à la coxa antérieure (photo 1).

- chez $\boldsymbol{E}$. dispar, le thorax est brun foncé, souvent rougeâtre, à peu près de la même couleur dorsalement et ventralement. Les pleures sont plus ou moins blanchâtres.

Les pattes antérieures sont plus foncées que les autres chez les deux espèces, mais les pattes d'une même paire sont en géuéral plus foncées chez $E$. dispar que chez $E$. pazsiczkyi.

Les ailes semblent présenter une différence caractéristique : chez $E$. pazsiczkyi, les nervures transversales sont plus foncées (brun nettement violacé) que les nervures longitudinales (brun clair). Ce n'est'pas le cas chez $E$. dispar. Ce caractère, très net, 
apparaît encore sur les individus de la collection DESPAX pourtant conservés en alcool depuis 1926 ot 1932.
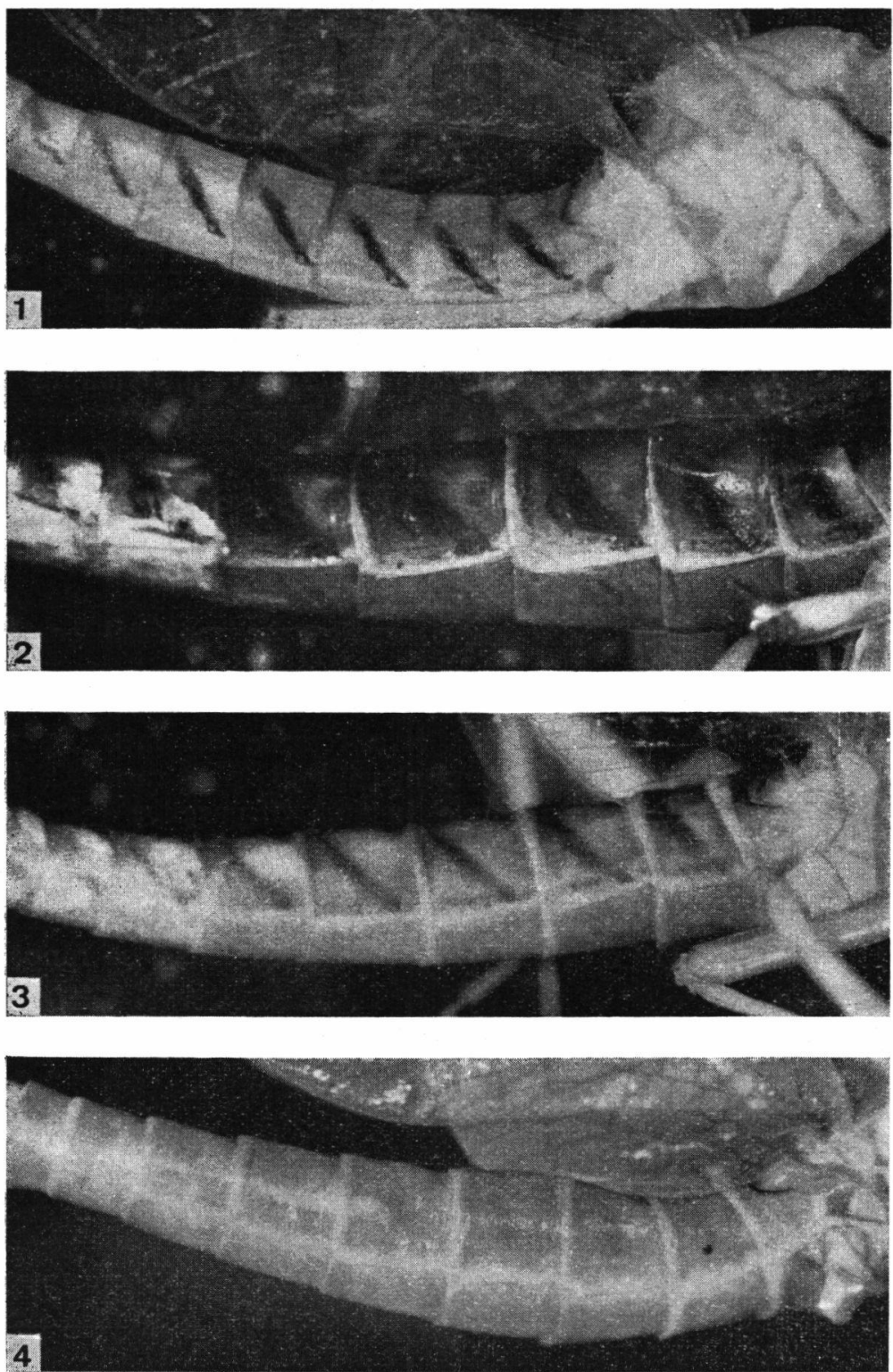

Photographies de l'abdomen d'Ecdyonurus (vues latérales).

1 : E. pazsiczki. 2 : E. dispar (exemplaire à taches larges). 3 : E. dispar (exemplaire à taches étroites). $4: E$. dispar (exemplaire dépourvu de taches). 
Abdomen :

- chez E. pazsiczkyi les tergites portent latéralement une bande étroite, oblique, sombre, violet intense à noire. Elle se dirige vers le spiracle, en s'amincissant progressivement, jusqu’à celui-ci, puis elle s'étale légèrement à nouveau au-delà du spiracle (photo 1 ).

La couleur des tergites est d'un jaune rosâtre translucide qui peut s'accentuer jusqu'à former une bande transversale brun cuivrée au bord postérieur des tergites, entre les bandes obliques. Cette couleur passe peu à peu à un jaune blanchâtre, souvent translucide, dans les coins antérieurs des tergites (près des spiracles), au-dessous des bandes obliques et sur les pleures.

Les sternites sont eux aussi blanc jaunâtres, parfois à peine cuivrés très pâles et plus ou moins translucides.

- chez $E$. dispar, la teinte dominante est plus sombre. Les tergites sont d'une couleur à peine plus claire que le mesonotum, cette teinte s'éclaircissant vers les pleures. Le brun cuivré prend souvent une nuance rougeâtre, ce qui est particulièrement visible sur la plus grande partie des sternites surtout dans leur partic postérieure.

Kimmins [1942] indique que l'abdomen d'E. dispar est dépourvu, en Grande-Bretagne, de taches latérales, triangles ou bandes obliques. La quasi totalité des individus de mon matériel porte au contraire des taches latérales bien visibles. Il s'agit en général d'une bande courbe brun rougeâtre (photo 2) plus ou moins large, dont la partie postérieure peut être réduite ou même parfois manquer (photo 3 ). La tache peut aussi, mais plus rarement dans les Pyrénées, s'estomper presque jusqu'à disparaître (photo 4). La concavité de la courbe peut au contraire se pigmenter à son tour et conduire à une large tache triangulaire. La photo 3 peut expliquer la figure 27 de Schoenemund [1930]. De même, les figures $118 \mathrm{a}$ et $\mathrm{b}$ et 119 d'ULMER [1929] semblent aussi se rapporter à E. dispar.

\section{Genitalia.}

- Styligère.

Dans sa clé de détermination des Ecdyonurus français, FonTaine [1964] sépare les imagos mâles des deux espèces grâce à la dent située sur la face interne de chaque prolongement du styligère (D. fig. 1 et 2 ). Ces dents sont «bien marquées et incurvées vers l'intérieur» chez $\boldsymbol{E}$. dispar (ce qu'avait d'ailleurs signalé Kımmins en 1942) alors que chez $E$. pazsiczki on trouve "deux saillies latérales arrondies, bien développées ». La différence entre les deux espèces porte aussi sur la taille de cette denticulation. Elle est plus importante chez $E$. dispar et apparaît comme un prolongement très plat, haut de 0,08 à $0,1 \mathrm{~mm}$; par contre, la dent du styligère d'E. 

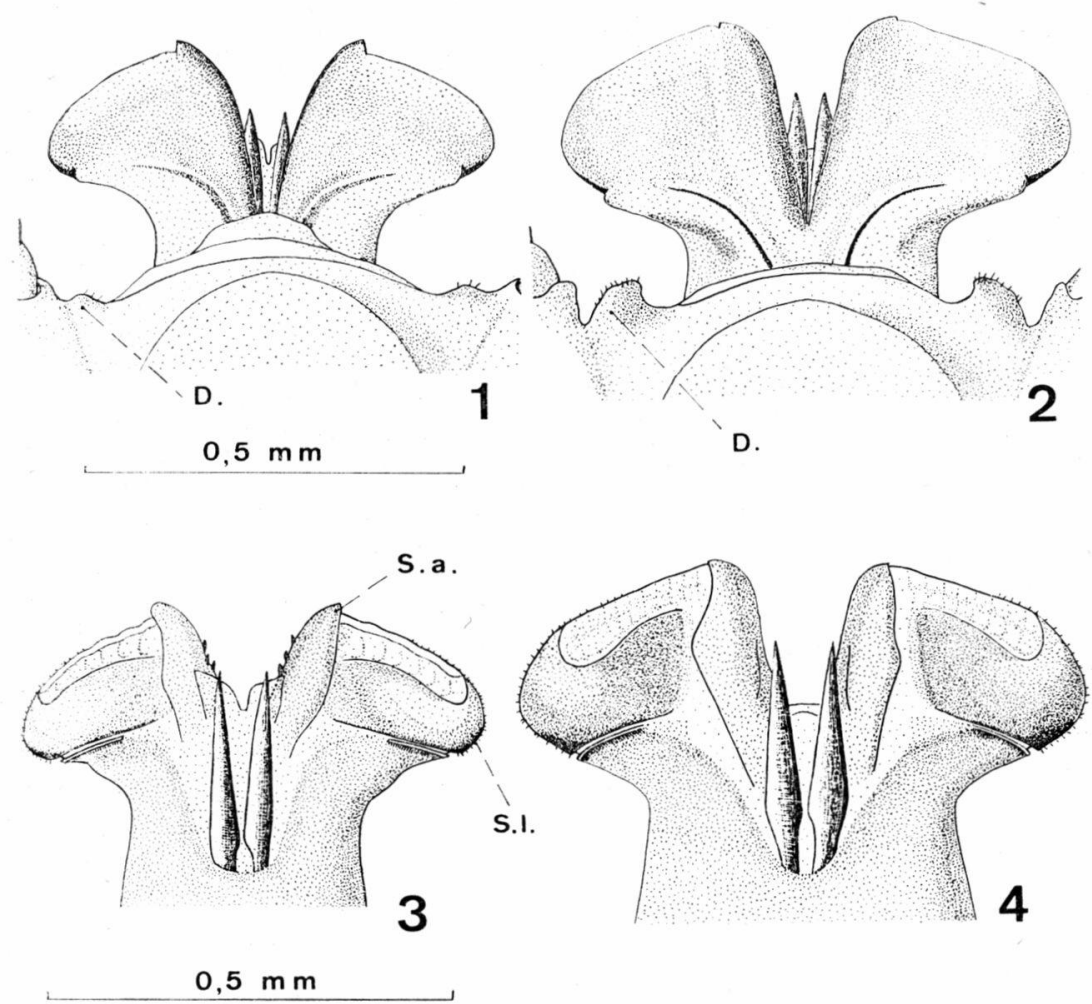

FIG. 1 à 4. - Genitalia mâles non éclaircis d'Ecdyonurus pazsiczkyi à gauche (fig. 1 et 3 ) et d'E. dispar à droite (fig. 2 et 4 ), d'exemplaires conservés en alcool. En haut (fig. 1 et 2 ) vues ventrales; $D=$ denticulation du styligère. En bas (fig. 3 et 4 ) vues dorsales; S. a. = sclérite apical, S. 1. = sclérite latéral.

pazsiczkyi mesure de 0,03 à $0,04 \mathrm{~mm}$ sur le matériel examiné jusqu'ici (fig. 1 et 2 ).

De plus, d'une manière générale, si la denticulation est effectivement recourbée vers l'intérieur chez $E$. dispar, ce caractère n'est pas constant [LANDA, 1959] ainsi qu'en témoigne le tableau suivant, dressé après l'examen d'une centaine d'imagos ô.

\begin{tabular}{|c|c|c|c|}
\hline $\begin{array}{c}\text { denticulation } \\
\pm \text { recourbée } \\
\text { encore } \\
\text { assymétrique } \\
\text { avec les 2 arêtes } \\
\text { rectilignes }\end{array}$ & $\begin{array}{c}\text { denticulation } \\
\text { symétrique }\end{array}$ & $\begin{array}{c}1 \text { denticulation } \\
\text { symétrique } \\
1 \text { denticulation } \\
\pm \text { recourbée }\end{array}$ \\
\hline 65 & 14 & 73 \\
\hline
\end{tabular}


- Pénis.

La principale différence concerne le sclérite latéral de chaque lobe. Observé par la face dorsale, ce sclérite est plus massif et plus large chez E. dispar (S. l. fig. 3 et 4 ). La concavité de son contour distal est aussi plus accusée. Le rapport de la longueur de ce sclérite à sa hauteur maximale est d'environ 2,3 à 3,0 chez $E$. pazsiczkyi (valeur moyenne 2,5) et de 1,4 à 1,7 chez $E$. dispar (valeur moyenne 1,5).

Les sclérites apicaux internes (S. a.) sont également un peu plus proéminents chez $E$. pazsiczkyi. Cependant on ne peut se baser entièrement sur ce dernier caractère. En effet, ces sclérites peuvent être plus ou moins saillants chez $E$. dispar selon la position exacte des sclérites latéraux et le mode de conservation de l'imago. Les muscles péniens plus ou moins contractés peuvent faire saillir différemment les sclérites apicaux internes.

\section{Remarque :}

Le rapport de la longueur du tarse de l'imago ồ à celle du tibia chez $E$. dispar est, d'après Kimmins [1942], de 1,8. Sur plus de 70 exemplaires du Sud-Ouest de la France, les valeurs relevées ont été les suivantes :

moyenne : 1,57 ( 1,54 et 1,55 pour les deux of anglais examinés), extrêmes : 1,20 et 1,86 .

Chez E. pazsiczkyi, ces valeurs sont respectivement de : 1,80 , $1,58,1,95$.

Ce caractère ne peut donc être retenu pour différencier les deux espèces.

\section{LARVES.}

- Expansions paranotales. 25 nymphes de chaque espèce ont été étudiées. FonTaine [1964], dans sa clé de détermination des larves, indique la différence suivante : la longueur des expansions latérales prothoraciques est contenue 4 fois et même 5 dans la longueur totale du prothorax chez E. pazsiczkyi s. n. fluminum et un peu plus de 3 fois chez $E$. dispar. Ce critère permet en effet de déterminer une grande partie des individus. Mais les expansions paranotales des Ecdyonurus offrent une variabilité assez importante et, sur 50 spécimens pris au hasard dans mon matériel, les chiffres suivants sont relevés :

$\begin{array}{lll}\text { E. pazsiczkyi : valeur moyenne }: 4,46 \\ & \gg & \text { minimale }: 3,78 \\ - & \gg & \text { maximale }: 5,83 . \\ \text { E. dispar : } & \gg \text { moyenne }: 3,46 \\ & \gg \text { minimale }: 2,90 \\ & \gg \text { maximale }: 4,00 .\end{array}$


Environ $20 \%$ des larves sont ainsi absolument indéterminables (valeurs du rapport comprises entre 4,00 et 3,78) et la détermination d'un pourcentage de larves encore important (valeurs du rapport peu $<3,78$ ou peu $>4,00$ ) reste douteuse.
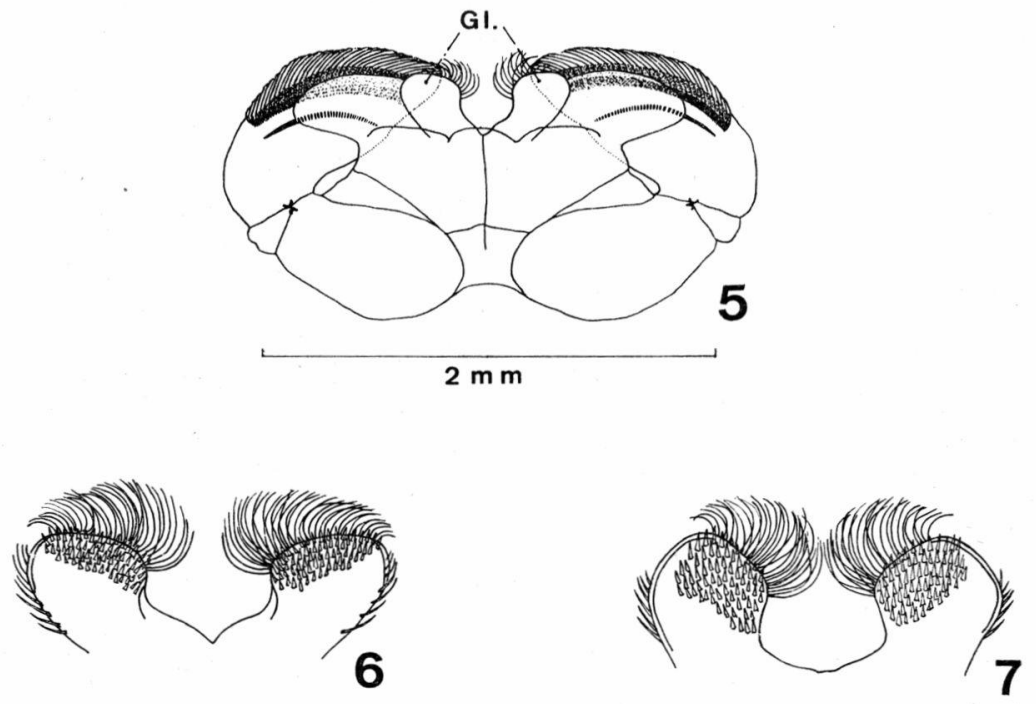

$1 \mathrm{~m} \mathrm{~m}$
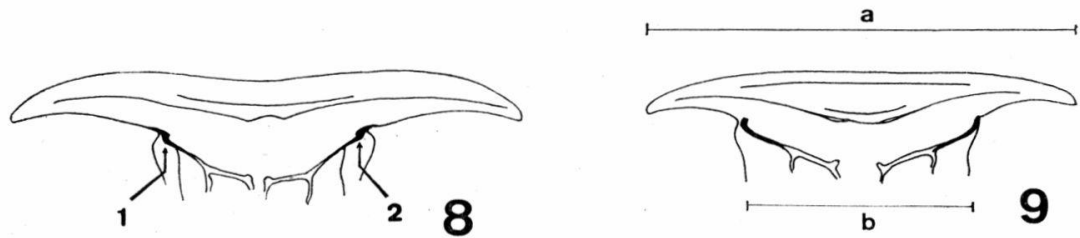

Fig. 5 à 9. - Appareils buccaux d'Ecdyonurus. Fig. 5 : labium d'E. pazsiczkyi; Gl.=glosses. Fig. 6 et 7 : glosses d'E. pazsiczkyi (fig. 6) et d'E. dispar (fig. 7 ). FIG 8 et 9 : labres d'E. pazsiczkyi (fig. 8) et d'E. dispar (fig. 9); 1 et 2 : épaississements cuticulaires déterminant la longueur b.

L'étude des appareils buccaux permet une distinction plus sûre de ces larves.

- Labium. Le contour apical des glosses (Gl. fig. 5) est à peu près rectiligne et presque perpendiculaire au plan de symétrie de la larve chez E. pazsiczkyi (fig. 6); il a une forme en «V》 renversé, «en toit», nettement marquée chez $E$. dispar (fig. 7).

Cette différence est constante pour le matériel examiné. 
- Labre. Un critère complémentaire peut aussi être utilisé. Chez les Ecdyonurus, le labre est relativement peu proéminent mais assez étalé latéralement. Chez $E$. pazsiczkyi, cet étalement latéral est beaucoup plus marqué que chez $E$. dispar. Le rapport de la largeur totale du labre à la largeur de son insertion ${ }^{1}$ est plus élevé chez $E$. pazsiczkyi $(2,50$ en moyenne) que chez $E$. dispar $(1,85)$. La différence est nette et perceptible sans recours à des mesures (sur les figures 8 et 9 : valeurs de 2,66 et 1,87 ).

La variabilité est importante mais aucun chevauchement entre les valeurs extrêmes des deux espèces n'a été encore observé. Les valeurs extrêmes sont de 2,22 et 2,86 chez $E$. pazsiczkyi et de 1,69 et 2,04 chez $E$. dispar. Ces mesures ont été effectuées directement sur les larves; des montages systématiques de labres entre lame et lamelle auraient certainement accru l'écart signalé. L'aplatissement a en effet tendance à redresser les extrémités du labre d'E. pazsiczkyi, souvent amincies et quelque peu enroulées vers l'intérieur.

\section{Remarque.}

La distinction des larves de ces deux espèces de celles d'E. venosus se fait aisément par la taille (en général nettement inférieure), la forme des expansions paranotales, et surtout par le nombre de denticulations des griffes tarsales.

Kimmins [1942], puis Macan [1949] ont montré chez les larves d'Ecdyonurus entre autres deux tendances : l'une à 2 denticulations par griffe (E. venosus), l'autre à 3 ou 4 ( $E$. torrentis et $E$. dispar). L'observation d'une centaine de pattes de chaque espèce conduit au tableau suivant :

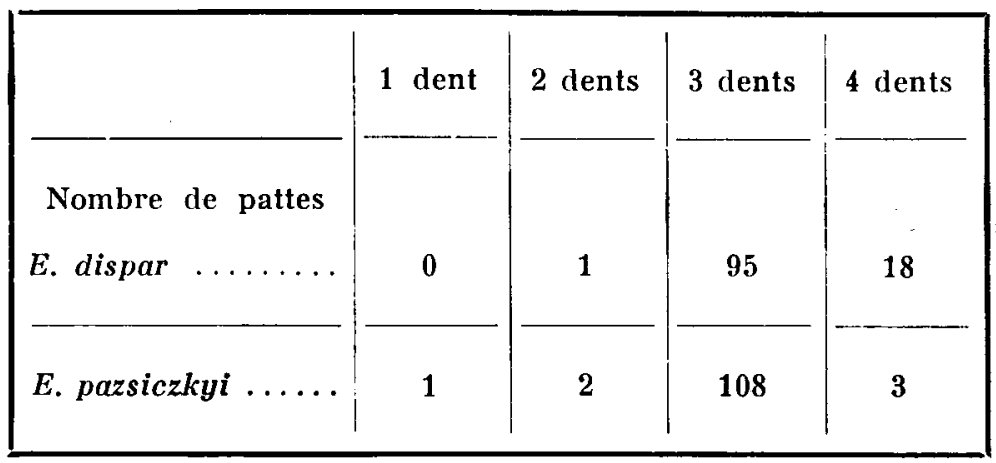

1. a/b (fig. 8 et 9 ) ; b étant mesuré entre les épaississements cuticulaires : flèches 1 et 2 (fig. 8). 


\section{3. - E. VENOSUS [FABRICIUS, 1775]}

ET E. FORCIPULA [PICTET, 1844]

L'examen de 70 imagos of d'E. forcipula et de 45 d'E. venosus -- dont deux exemplaires anglais -, en grande partic accompagnés de leurs exuvies larvaires, montre qu'il s'agit de deux espèces distinctes mais très voisines et qui ont sans doute été souvent confondues.

\section{1. - Matériel examiné :}

- E. forcipula :

Hautes-Pyrénées : Massif de Néouvielle :

vallon d'Estibère : Lac Supérieur et son déversoir à $2330 \mathrm{~m}$ (22-VIII-1962;

H. DÉcamps leg.; 5-25-VIII-1965 : A. T. leg.); Lac de l'Ile à $2280 \mathrm{~m}$ (20-VII-1961 :

H. Décamps leg.; 7-20-IX-1965 : A. T. leg.).

vallon de Port-Bieil : Grand Lac de Port-Bieil à $2280 \mathrm{~m}$ (25-VII-1960 : C.

BerthéLEMY leg.); Lac du Gourguet à $2220 \mathrm{~m}$ (9-VIII-1966 : H. Laville leg.);

Lac du Gourg de Nère moyen à $2200 \mathrm{~m}$ (18-IX-1966 : M $\mathrm{M}^{\text {me }}$ J. REY leg.); R. des

Pins à $1900 \mathrm{~m}$ (18-IX-1967 : A. T. leg.).

Neste de Couplan et affluents, de 1600 à $1200 \mathrm{~m}$ (21-VI à 25-VIII-1965 : A. T. leg.). leg.).

Déversoir du Lac de Bordères-Louron vers $1500 \mathrm{~m}$ (5-25-VIII-1965 : A. T.

一 E. venosus :

Ariège : l'Arize à $300 \mathrm{~m}$ (4-X et $13-\mathrm{XI}-1963,10-\mathrm{VI}$ et $18-\mathrm{VII}-1964,20-\mathrm{VII}$ et 21-X-1965 : C. BerthéLEMy leg.); le Lez à $430 \mathrm{~m}$ (IX et X-1959, V-IX-1962, VII-IX-1963, VII-1965 : C. BenthéLEMY leg.).

Cantal : vallée de la Jordanne (23-V-1964 : H. Décamps leg.).

\section{2. - Comparaison des deux espèces.}

Adultes.

Coloration :

- chez $E$. venosus, la coloration semble assez constante. Une couleur dominante, rouge vineux, et d'autant plus nette que l'imago est âgé ${ }^{1}$, se retrouve à peu près sur tout le corps.

Thorax brun rougeâtre. Membranes articulaires des ailes roses à rouges. Abdomen avec une double ligne dorsale médiane rouge, au moins sur les deux ou trois premiers tergites. Couleur générale de l'abdomen rose à brun rougeâtre. Les taches latérales, larges, triangulaires, sont d'un rouge vineux intense; les parties centrale et inférieure de la tache sont un peu plus claires, de sorte qu'une "arche» est visible.

1. Sur les individus capturés peu après l'abandon de l'exuvie subimaginale, la coloration est bien plus claire, aussi bien le fond que les taches latéroabdominales. Dans le cas d'imagos obtenus par élevage, il est préférable de laisser «survivre» les individus pendant 24 heures environ dans un bocal grillagé avant leur mise en alcool. 
- chez E. forcipula, la coloration paraît au contraire très variable. D'une façon générale, elle est bien moins rouge que chez E. venosus.

Les taches latérales des individus de la collection MAC LACHLAN ( «E. fluminum variété foncée») sont d'un brun fauve foncé. Sur mes exemplaires, elles peuvent varier de brun foncé à bistre diffus, et dans ce dernier cas, très étendues, elles finissent par se noyer dans la coloration dorsale des tergites. Elles peuvent être aussi, rarement, rougeâtres ou violacées. Sur les nombreux exemplaires de cette espèce capturés dans la vallée de la Neste d'Aure, il n'y a pas de taches ou bien ces taches, très estompées, se devinent à peine. L'abdomen est alors d'un jaune foncé uniforme, parfois légèrement ocré, qui s'intensifie un peu vers le bord postérieur médian des tergites. Le brun foncé du thorax ne varie guère.

La forme décrite par Pictet en 1844 sous le nom "Baetis montana » est peut-être un Ecdyonurus forcipula peu coloré.

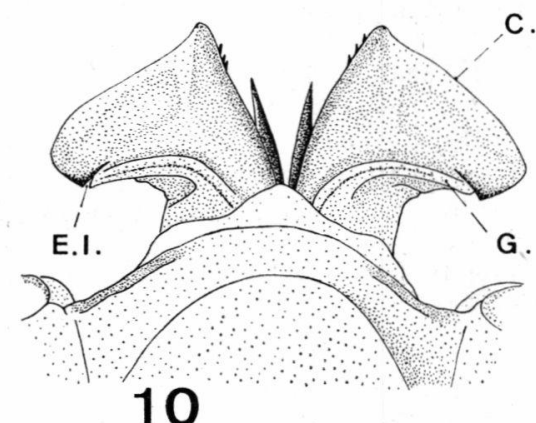

10

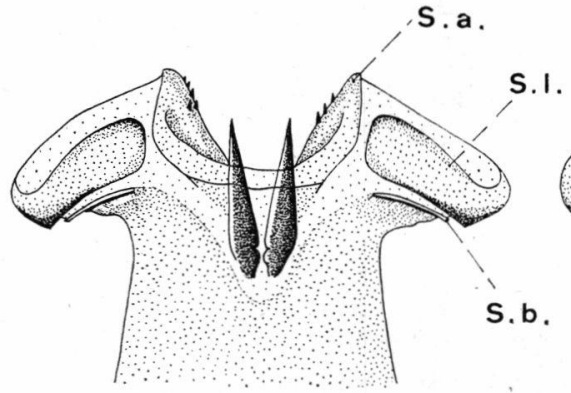

12

C.a.

S.I.

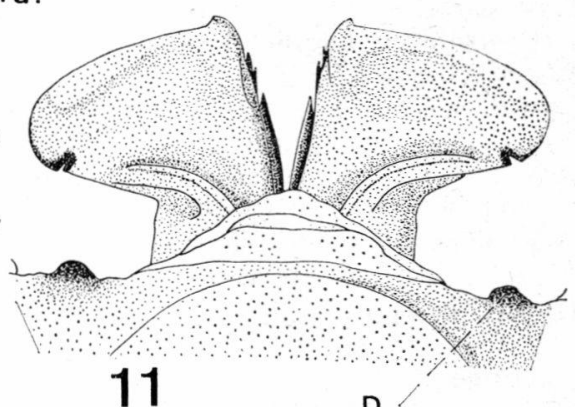

11

D.

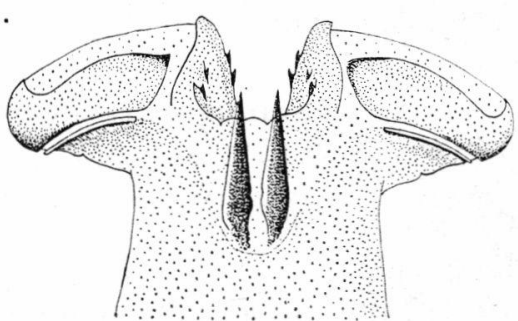

13

FIG. 10 à 13. - Genitalia mâles non éclaircis d'E. venosus à gauche (fig. 10 et 12 ) et d'E. forcipula à droite (fig. 11 et 13 ) d'exemplaires conservés en alcool. En haut, vues ventrales (fig. 10 et 11 ); E. l. = encoche latérale, C. a. = contour apical des lobes, G. = gouttière, D. = denticulation du styligère. En bas, vues dorsales (fig. 12 et 13 ); S. a. = sclérite apical, S. $1 .=$ sclérite latéral, S. b. = sclérite basal. 
Cela n'est pas sans rappeler les variations de coloration abdominale d'E. dispar, dépourvu de taches latéro-abdominales, au moins en Grande-Bretagne.

\section{Genitalia.}

Les genitalia de ces deux espèces sont très proches et leur grande variabilité de forme tend, dans les cas extrêmes, à les rapprocher encore. La détermination d'animaux décolorés, en l'absence d'exuvie nymphale, peut à la limite devenir très difficile et même incertaine.

On remarque cependant plusieurs petites différences qui, sans être tout à fait constantes, permettent, dans la majorité des cas, la distinction des deux espèces uniquement sur l'examen du styligère et des lobes péniens.

\section{— Styligère.}

Kimmins [1942] a signalé que le styligère d'E. venosus est, en principe, dépourvu de denticulation à l'angle interne des styles. Mes résultats confirment ce fait pour les Pyrénées, avec toutefois une certaine variabilité, comme en témoignent les chiffres suivants :

pas de denticulation nette sur 39 ô (fig. 10), une denticulation petite mais visible sur $6 \hat{o}^{1}$.

Chez $E$. forcipula, en général, on trouve au contraire, des denticulations nettes, de couleur bistre :

denticulation nette sur 61 \& (D. fig. 11), denticulation inexistante ou très réduite sur 7 s.

\section{- Lobes du pénis.}

Ils ont une forme assez voisine chez les deux espèces. Cependant, en général, le contour apical des deux lobes forme un $《 V »$ un peu plus fermé chez $E$. venosus, avec des sclérites apicaux un peu plus saillants (C. a., S. a., fig. 10 à 13). Le bord externe des sclérites latéraux (S.l.) est plus régulièrement arrondi chez $E$. forcipula; chez $E$. venosus, il est souvent rectiligne à la base, et se recourbe ensuite seulement vers les sclérites apicaux. Les sclérites latéraux surplombent davantage le sclérite basal (S. b.) à l'extrémité chez $E$. venosus. Cette disposition est souvent visible en vue dorsale et même ventrale, où l'encoche latérale (E. l.) ainsi formée peut être un peu plus longue chez E. venosus. Toujours en vue ventrale, la double gouttière (G.), dont une moitié se continue sur chaque lobe, se poursuit souvent plus loin latéralement chez $E$. venosus et se recourbe davantage, à son extrémité distale, vers

1. Il n'est pas tenu compte du bourrelet allongé assez fréquent qui correspond à un léger épaississement du styligère à cet endroit (fig. 10). 
le styligère. Ces caractères sont perceptibles sur des pénis non éclaircis. En effet, la disparition des muscles péniens, par traitement à la potasse par exemple, tend le plus souvent à réduire les différences signalées.

Remarque :

Des mesures systématiques entreprises sur les pattes antérieures des individus mâles (rapport de la longueur du tarse à celle du tibia) n'apportent aucun caractère distinctif supplémentaire. Tout au plus, semble-t-il que la variabilité soit plus faible chez $E$. venosus, malgré un nombre supérieur de spécimens examinés.

\begin{tabular}{|c|c|c|c|}
\hline & $\begin{array}{c}\text { Valeur moyenne } \\
\text { du rapport } \\
\text { de la longueur du tarse }\end{array}$ & \multicolumn{2}{|c|}{ Valeurs extrêmes } \\
\hline $\begin{array}{l}\text { E. forcipula } \\
30\end{array}$ & 1,51 & 1,76 & 1,29 \\
\hline $\begin{array}{l}\text { E. venosus } \\
45 \text { a }\end{array}$ & 1,51 & 1,77 & 1,36 \\
\hline
\end{tabular}

Si l'on retire de ces deux séries les quatre individus assez exceptionnels correspondant aux valeurs extrêmes, les nouvelles valeurs extrêmes, beaucoup plus significatives, deviennent 1,66 et 1,30 pour $E$. forcipula, 1,64 et 1,41 pour $E$. venosus.

\section{LARVES.}

Au stade nymphal, elles se distinguent facilement grâce aux fortes épines qui prolongent latéralement et ventralement chaque segment abdominal. La taille de ces épines varie selon le segment considéré (taille maximale pour les segments 4 et 5 en général) et selon les individus d'une même espèce. Cependant, on remarque que les épines sont dirigées vers l'extérieur chez $E$. venosus et, au contraire, plus ou moins nettement vers l'intérieur chez $E$. forcipula (fig. 14 et 15). La longueur de ces épines est, pour les segments 4 et 5 , de l'ordre du tiers de la longueur du segment abdominal correspondant chez $E$. venosus, et de l'ordre du $1 / 5^{\circ}$ ou du $1 / 6^{*}$ seulement chez $E$. forcipula.

D'autre part, MacaN [1949] a mis en évidence la variabilité présentée par le nombre de denticulations des griffes tarsales chez $E$. venosus. En général, on compte cependant deux dents par griffe. 
Mes mesures confirment pour les Pyrénées les chiffres donnés par MACAN, et on peut dire qu'environ un individu sur deux porte une ou deux pattes avec trois dents, les autres pattes étant porteuses de deux dents. Chez $E$. forcipula au contraire, le nombre de denticulations est beaucoup plus constant : sur 120 pattes examinées, deux seulement sont porteuses de trois dents (la troisième très réduite) et appartiennent d'ailleurs à la même nymphe; toutes les autres portent deux dents.

\section{4. - ECDYONURUS ANGELIERI N. SP. (=E. forcipula Navas pro parte)}

Des élevages de larves capturées principalement dans la Neste de Couplan (Hautes-Pyrénées), lors de l'été 1965, ont permis d'obtenir une espèce nouvelle du genre Ecdyonurus que je dédie à M. le Professeur Angelier.

L'examen des Ecdyonurus d'une partie de la collection Navas," conservée au Musée de Zoologie de Barcelone, montre que cette espèce, assez répandue dans les Pyrénées, a déjà été trouvée en Espagne et désignée par Navas par le nom d'E. forcipula. Ces deux espèces sont voisines mais l'examen des genitalia des imagos mâles en permet une distinction aisée.

Ma collection compte plus de 80 imagos mâles très souvent accompagnés de leurs exuvies larvaires et provenant des cours d'eau suivants : Neste de Couplan, Neste d'Aure (Hautes-Pyrénées), Massane (Pyrénées-Orientales), Lez (Ariège), quelques exemplaires provenant en outre des Basses-Pyrénées. Deux spécimens de la collection Navas et provenant de Catalogne ( $S^{t}$ Privat et Espluga de Francoli) ont également été examinés.

\section{1. - Imago mâle.}

\section{Coloration.}

Yeux de teinte générale bleu foncé à reflets superficiels blanchâtres accusés et dus aux cornéules. Parallèlement au bord inférieur et extérieur de l'œil, plusieurs stries se succèdent:

- une ligne blanchâtre très fine fait suite au fond bleu,

- une bande bleu-violacée tirant parfois sur le rose,

- une bande blanchâtre large.

Ocelles volumineux cerclés de deux anneaux concentriques, l'extérieur brun foncé, l'intérieur brun clair.

Thorax dorsalement brun moyen (angle postérieur médian brun noirâtre), ventralement brun plus foncé, pleures transparentes ou blanchâtres. Pattes antérieures brunes, parfois rougeâtres, s'éclaircissant au niveau des tarses. Pattes postérieures gris-jau- 
nâtres, les tarses au contraire plus foncés, d'un brun moyen, de même que l'articulation fémur-tibia.

Ailes à nervures longitudinales et transversales brunes ; costale, sous-costale et radiale souvent plus foncées. Aire ptérostigmatique bien visible, blanc-jaunâtre parfois légèrement brune.

Abdomen dorsalement châtain clair, la couleur devenant plus foncée vers le bord postérieur des tergites, et plus claire à la fois
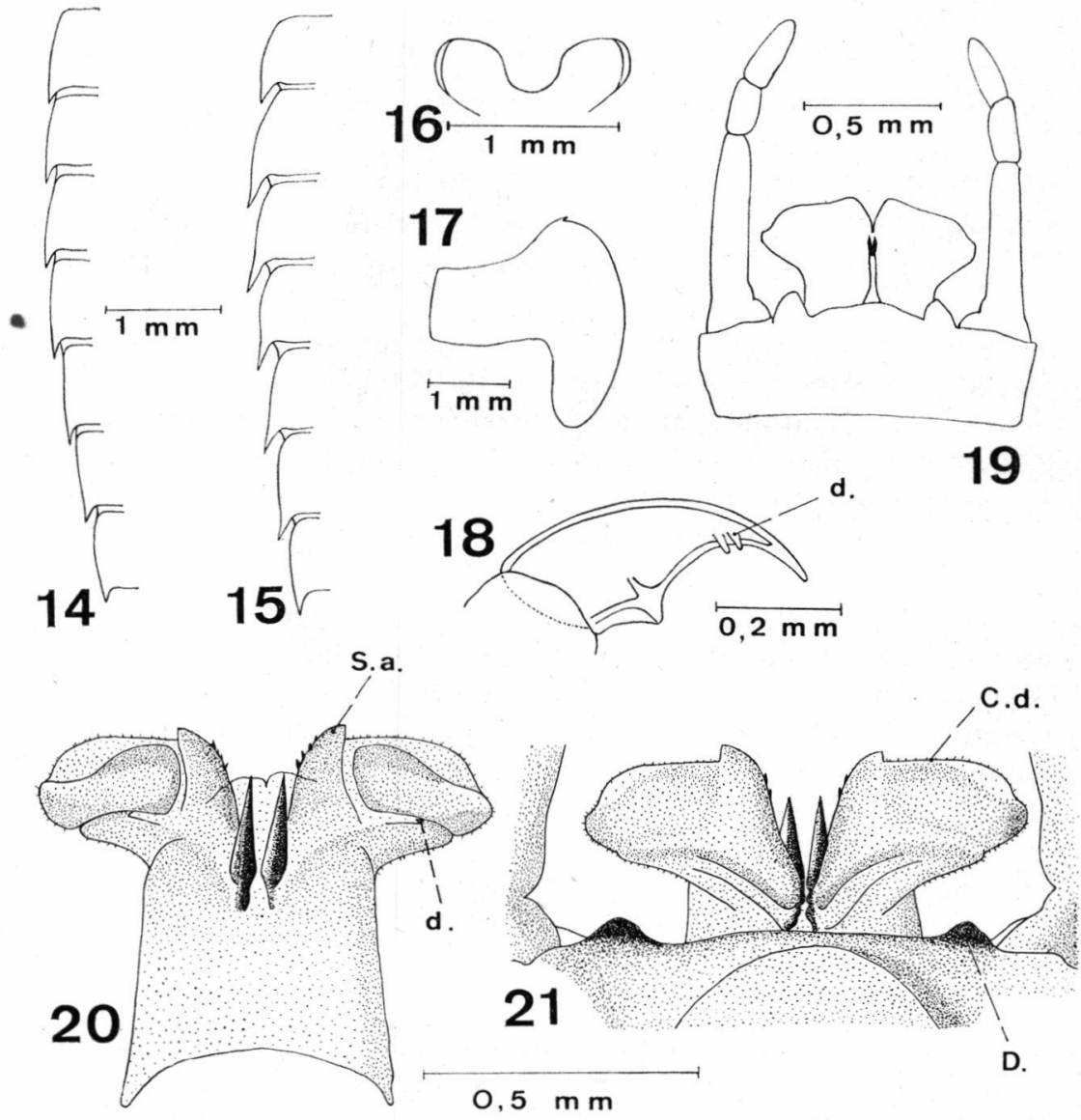

19

FIG. 14 et 15 : contour latéral de l'abdomen de nymphes d'Ecdyonurus en vue ventrale; fig. 14 chez $E$. forcipula et $E$. angelieri n. sp., fig. 15 chez $E$. venosus.

Fig. 16 à 18 : E. forcipula et E. angelieri n. sp.; fig. 16 : glosses; fig. 17 : demipronotum ; fig. 18 : griffe tarsale, d.=denticulation.

FIg. 19 à 21 : E. angelieri n. sp. (exemplaires conservés en alcool); fig. 19 : styligère, styles et pénis du subimago $\sigma^{7}$; fig. 20 : pénis non éclairci en vue dorsale, $\mathrm{S}$. a. = sclérite apical, d. = denticulation du sclérite basal; fig. 21 : genitalia non éclaircis en vue ventrale, C. d. = contour distal des lobes, D. = denticulation du styligère. 
vers leur bord antérieur et vers les pleures. Une double bande médiane, étroite et brun rouge, parcourt parfois les tergites. Latéralement, les tergites 2 à 8 sont traversés par une bande oblique large, légèrement incurvée et de couleur brun rouge. Une zone tranparente surmonte l'extrémité de cette bande, au-dessus du spiracle. Pleures blanc-jaunâtres. Les sternites portent dans leur partie postérieure une tache polygonale rougeâtre.

Cerques brun foncé à la base, s'éclaircissant progressivement jusqu'à une teinte chair à l'extrémité postérieure.

\section{Genitalia.}

Styligère et moitié antérieure des styles brun foncé à très foncé, moitié postérieure brun-gris clair. Denticulations du styligère bistres, larges et bien visibles (D. fig. 21).

Pénis brun cuivré clair.

Épaississement interne du sclérite basal terminé de chaque côté par une ou deux denticulations dorsales fortement chitinisées. Les sclérites latéraux, assez allongés et fortement renflés dans leur moitié interne, s'amincissent progressivement vers l'extérieur par une longue courbe : l'élargissement terminal des sclérites latéraux est peu marqué.

Les deux caractères les plus significatifs opposant notamment cette espèce à $E$. forcipula sont les suivants :

- les deux sclérites apicaux, écartés, sont en général très proéminents et nettement incurvés vers l'extérieur en une courbe continue (S. a., fig. 20),

- le contour distal des lobes du pénis qui, au-delà des sclérites apicaux, se poursuit perpendiculairement au plan de symétrie de l'animal pour rejoindre le sclérite latéral par une courbure fortement proéminente (C. d., fig. 21).

Le sclérite basal, les sclérites latéraux et le contour distal des lobes du pénis sont hérissés de poils courts et épais plus ou moins épineux. Les sclérites apicaux portent 6 à 8 fortes épines courtes, plus ou moins recourbées, et dirigées vers les cerques de l'animal.

En vue ventrale, la surface des lobes du pénis forme deux gouttières profondes dirigées vers l'extérieur. Les deux titillateurs sont assez minces et coniques dans leur partie apicale.

\section{2. — Imago femelle.}

La femelle est plus claire que le mâle.

Thorax dorsalement jaune noisette à brun très clair. Pattes et nervation un peu plus claires que chez le mâle.

Abdomen à bandes longitudinales et obliques rougeâtres sur un fond jaunâtre. Taches des sternites moins prononcées que chez le mâle. Plaque sous-génitale peu caractéristique, progressi- 
vement arrondie, ne dépassant pratiquement pas le contour apical du dernier tergite.

\section{3. - Subimago mâle.}

Tergites abdominaux 10,9 , et même parfois 8 , plus foncés que les précédents. Taches latéro-abdominales plus ou moins visibles, d'un brun grisâtre.

Styles plus courts et plus épais que chez l'imago. Denticulations du styligère fortes, arrondies au sommet, souvent asymétriques (fig. 19). Le contour latéro-distal du pénis est moins nettement perpendiculaire au plan de symétrie que chez l'imago. Le contour interne des sclérites apicaux, déjà nettement arrondi, constitue l'élément le plus caractéristique. Il permet de séparer le subimago d'E. angelieri de ceux des espèces du groupe venosus dont les sclérites apicaux sont nettement plus contigus sur leur face interne.

\section{4. - Larve.}

Elle appartient au groupe venosus. Il n'a pas été possible jusqu'à présent de la distinguer de la larve d'E. forcipula.

Les épines latéro- abdominales permettent done de la séparer de celle d'E. venosus (fig. 14).

La présence de deux denticulations sous chaque griffe tarsale est très constante et éloigne $E$. angelieri d'E. wautieri Fontaine 1964 (fig. 18). Les expansions paranotales sont également plus longues que chez $E$. wautieri (fig. 17), le rapport de la longueur totale d'une expansion, mesurée de l'épine antérieure au bord postérieur, à la largeur maximale du demi-prothorax étant de 1 (0,8 sur la fig. 9 de Fontaine 1964).

L'holotype $\delta$, l'allotype $\&$ et une vingtaine de paratypes sont conservés en alcool à $80^{\circ}$ dans ma collection, au laboratoire de Zoologie de Toulouse. Ils proviennent tous de la Neste de Couplan à Artigusse et ont été capturés le 20-VII-1967.

Au point de vue écologique, $E$. wautieri est fréquent dans le Rhône à Lyon [Fontaine, 1964]. E. angelieri est une espèce de moyenne altitude en Vallée d'Aure; elle est fréquente dans les ruisscaux et petits cours d'eau peu pollués entre 500 et $1600 \mathrm{~m}$. $E$. forcipula cohabite avec $E$. angelieri dans la Neste de Couplan, vers $1200-1600 \mathrm{~m}$. Mais $E$. forcipula remonte jusqu'à $2300 \mathrm{~m}$ d'altitude; dans le massif de Néouvielle elle a été récoltée dans des torrents et des lacs de l'étage subalpin. 


\section{5. - CLE DE DETERMINATION DES ECDYONURUS DU SUD-OUEST DE LA FRANCE}

\section{1. — Imagos mâles.}

1. Sternites abdominaux présentant trois taches allongées et deux taches punctiformes disposées en «abat-jour de lampe»....... insignis.

- Sternites abdominaux ne présentant pas cette ornementation .... 2 .

2. Ailes à nervures tranverses violet foncé, ̀̀ nervures longitudinales brunes. Abdomen blanc jaunâtre à bandes obliques, étroites, violet très intense ou noires. (Genitalia fig. 1 et 3 ) ....... pazsiczkyi.

- Ailes à nervures tranverses brunes. Abdomen rouge vineux, brun rougeâtre ou brun jaunâtre, à taches latérales larges, en général en forme de «pont» ou de triangle, parfois absentes ........... 3 .

3. Lobes du pénis relativement étroits, de forme assez équilatérale; dents du styligère proéminentes, le plus souvent incurvées vers l'inté-

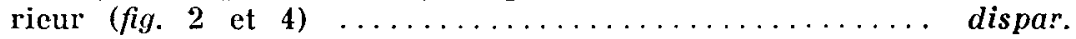

- Lobes du pénis fortement étalés latéralement; dents du styligère absentes ou relativement peu développées .............. 4 .

4. Lobes du pénis à contour distal perpendiculaire au plan de symétrie de l'animal; contour des sclérites apicaux internes régulièrement convexe (fig. 20 et 21$) \ldots \ldots \ldots \ldots \ldots \ldots \ldots \ldots \ldots \ldots \ldots \ldots \ldots \ldots \ldots$ angelieri.

-- Lobes du pénis formant un angle plus ou moins obtus; sclérites apicaux internes non régulièrement convexes (fig. 12 et 13) $\ldots .5$.

5. Couleur de l'abdomen nettement rougeâtre; en général, pas de denticulations sur le styligère $(f i g, 10) \ldots \ldots \ldots \ldots \ldots \ldots$ venosus.

- Couleur du corps brun rougeâtre à brun jaunâtre, taches latéroabdominales parfois absentes. En général, des denticultions bien visibles à l'angle interne des styles (fig. 11) ........... forcipula.

\section{2. - Larves.}

1. Toutes les branchies portent une touffe de filaments trachéens .....

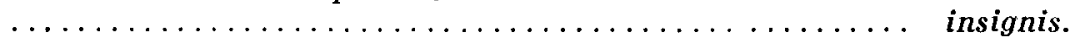

- Seules les branchies des 6 premières paires portent une touffe de

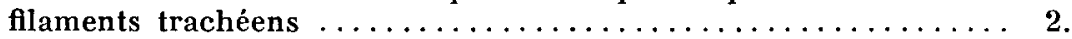

2. Deux denticulations (rarement trois) à chaque griffe tarsale; expansions latérales prothoraciques de grande taille . . . . . . . . . 3 .

- Trois denticulations (parfois quatre) à chaque griffe tarsale; expansions latérales prothoraciques de taille relativement réduite .....4 4 .

3. Epines latéro-abdominales en général longues $(1 / 3$ de la longueur du segment correspondant) et dirigés vers l'extérieur (fig. 15)

venosus

- Épines latéro-abdominales en général relativement courtes $(1 / 5$ à $1 / 6$ de la longueur du segment abdominal correspondant) et dirigées légèrement vers l'intérieur ou parallèles à l'axe du corps (fig. 14) . . $\ldots \ldots \ldots \ldots \ldots \ldots \ldots \ldots \ldots \ldots \ldots \ldots \ldots \ldots \ldots$ forcipula ou angelieri.

4. Labium portant des glosses à contour apical en « toit», en « V » renversé (fig. 7) $\ldots \ldots \ldots \ldots \ldots \ldots \ldots \ldots \ldots \ldots \ldots \ldots \ldots \ldots \ldots \ldots \ldots$ dispar.

- Labium portant des glosses à contour apical presque rectiligne et perpendiculaire au plan de symétrie $(f i g .6) \ldots \ldots \ldots$ pazsiczkyi. 


\section{RÉSUMÉ}

Les synonymies $E$. fluminum Pictet $=E$. dispar Curtis et $E$. fluminum Eaton (pro parte) $=E$. forcipula Pictet sont proposées.

La «variété claire 》d' $E$. fluminum introduite par EATon est une espèce distincte; elle est appelée ici E. pazsiczkyi Pongracz à la suite de la synonymie proposée par Illies [1967].

Les espèces $E$. pazsiczkyi et $E$. forcipula sont redécrites et opposées respectivement à $E$. dispar et $E$. venosus.

E. angelieri n. sp. des Pyrénées est décrite aux trois stades : adulte, subimaginal et larvaire.

Une clé de détermination des adultes et des larves des Ecdyonurus du Sud-Ouest de la France est présentée.

\section{THE TAXONOMY OF SOME ECDYONURUS OF THE SOUTH-WEST OF FRANCE}

The synonymies $E$. fluminum Pictet $=E$. dispar Cuntis and E. fluminum Eaton (pro parte) $=E$. forcipula Pictet are proposed.

The light variety of $E$. fluminum introduced by EAToN is a distinct species; here it is called E. pazsiczkyi, after the synonymy proposed by ILLIES [1967].

The species $E$. pazsiczkyi and $E$. forcipula are described a second time and are compared respectively with $E$. dispar and $E$. venosus.

$E$. angelieri $\mathrm{n}$. sp. from the Pyrenees is described in three stages : adult, subimago and larval.

A key for the determination of the adults and of the larvae of the Ecdyonurus of the South-West of France is given.

\section{UBER DIE TAXONOMIE EINIGER ECDYONURUS AUS DEM SUD-WESTEN FRANKREICHS}

Es wird vorgeschlagen den $E$. fluminum Pictet $=E$. dispar Cuntis und $E$. fluminum Eaton (pro parte) $=E$. forcipula PICTET als Synonyme gleich zu setzen.

Die « light specimens» von $E$. fluminum, die durch EAToN eingeführt wurde, ist eine eigene Art; der von ILures [1967] vorgeschlagenen Synonymie folgend wird sie hier $E$. dispar und $E$. venosus genannt.

Die Arten $E$. pazsiczkyi und $E$. forcipula werden wieder neubeschrieben und sowohl der Art $E$. dispar als auch $E$. venosus gegenüber gestsellt.

Von $E$. anglieri n. sp. aus den Pyrenäen werden drei stadien : imaginal, subimaginal und Larvenstadium beschrieben.

Eine Bestimmungstabelle für die Imagos und die Larven des Ecdyonurus aus lem Süd-Westen Frankreichs ist angefügt. 


\section{TRAVADX CITÉS}

Bogoescu (C.). 1958. - Ephemeroptera. In Fauna Republicii Populare Romine, 7 (3) : 189 p. Bucuresti.

Bogoescu (C.) et Tabacaru (I.). 1962 - Beiträge zur Kenntnis der Untersuchungsmerkmale zwischen den Gattungen Ecdyonurus und Heptagenia (Ephemeroptera). Beitr. Ent., Berlin, 12 (3/4) : 273-292.

Cuntrs (J.). 1834. - Descriptions of some non descript British species of Mayflies. Lond. Edinb. Dubl. Phil. Mag. ser., 3 : 120-2.

Eaton (A. E.). 1871. - A Monograph on the Ephemeridae. Trans. $R$. Ent. Soc., London : $1-164+6 \mathrm{pl}$.

Eaton (A. E.). 1883-1888. - A Revisional Monograph of Recent Ephemeridae or Mayflies. Trans. Linn. Soc. Lond., Zoology, 3 : 1-352 $+65 \mathrm{pl}$.

Fontarne (J.). 1964. - Ecdyonurus wautieri sp. n., espèce nouvelle d'Heptageniidae rencontrée dans la région lyonnaise (Ephéméroptère). Bull. Soc. Linn. Lyon, 33 (3) : 84-91.

Grandr (M.). 1953. - Contributi allo studio degli Efemeroidei italiani. XVII. Ecdyonuridae. Boll. Ist. Ent. Univ. Bologna, 19 : 307-386.

Grandi (M.). 1960. - Ephemeroidea. In Fauna d'Italia, 3 : 472 p. Bologna.

Illies (J.) et coll. - Limnofauna Europaea. Stuttgart. xv +474 p.

Kimmins (D. E.). 1942. - The British species of the genus Ecdyonurus (Ephemerptera). Ann. Mag. nat. Hist., London, 11 : 486-507.

Krmmins (D. E.). 1954. - A revised Key to the adults of the British species of Ephemeroptera with notes on their ecology. Scient. Publs Freshwat. biol. Ass., 15 : 1-71.

Kimmins (D. E.). 1958. -- The Ecdyonurus helveticus (Eaton) Complex (Ephemeroptera). Ann. naturh. Mus., Wien, 62 : 225-232.

KuApalek (F.). 1909. - Ephemerida, Eintagsfliegen. In Die Süsswasserfauna Deutschlands, 8 (1) : $32 \mathrm{p}$. Jena.

I.ANd (V.). 1959. - Ephemeroptera. In Klíč $k$ fauně ČSR. 3 : 143-167, Praha.

Lestage (J. A.). 1923. - Catalogue des Ephémères de France. Annls Soc. ent. Fr., 91 (1922) : 273-276.

Macan (T. T.). 1949. - The taxonomy of the British species of the genus Ecdyonurus (Ephem.). Entomologist's mon. Mag., London, 85 : 64-70.

Mikuiski (J. S.). 1936. - Jetki (Ephemeroptera). In Fauna Stodkowodna Polski, 15 : 168 p. Warszawa.

PICTET (F. J.). 1844. - Histoire Naturelle générale et particulière des Insectes Névroptères. Scconde monographic : famille des Éphémérines. Genève. $1-300+47 \mathrm{pl}$.

Pongracz (S.). 1913. - Újabb adatok Magyarország. Neuroptera-fauná jához. Rovart. lap., 20 : 175-186, Budapest.

Schoenemund (E.). 1930. - Eintagsfliegen oder Ephemeroptera. In Die Tierwelt Deutschlands, 19 : 106 p. Jena.

Ulder (G.). 1929. - Ephemeroptera. In Die Tierwelt Mitteleuropas, 4 : 43 p. Leipzig.

(Laboratoire d'Hydrobiologie de la Faculté des Sciences de Toulouse Equipe de recherche associée au C.N.R.S.) 